\title{
Efficacy of closed loop feedback system with augmented virtual reality visual cues training on gait and functional performance in stroke patients
}

\author{
Abu Shaphe ${ }^{*}$, Iftikar Hussain Shalla ${ }^{2}$, Raid Saleem Al Baradie ${ }^{3}$ and Mohammad Qasheesh 4 \\ ${ }^{1}$ Associate Professor, Department of Physical Therapy, College of Applied Medical Sciences, Jazan University, \\ Jazan, Saudi Arabia \\ ${ }^{2}$ Senior Physiotherapist-2, ECU/Health Affair Department, Dubai Health Authority, Dubai, UAE \\ ${ }^{3}$ Associate Professor, Medical Lab Department, College of Applied Medical Sciences, Majmaah University, Al \\ Majmaaj, Saudi Arabia \\ ${ }^{4}$ Assistant Professor and Head Department of Physical Therapy, College of Applied Medical Sciences, Jazan \\ University, Jazan, Saudi Arabia
}

\section{ABSTRACT}

The traditional gait training techniques, which are not based on principles of motor control lack requisites in terms of training intensity, duration and enough practice to have any meaningful carryover effect. Therefore, the aim of this study is to investigate the efficacy closed loop visual cues incorporated augmented reality environment on functional gait and community ambulation in stroke patients. A randomized control trial with control group was designed and 14 subjected were recruited in each group. Four weeks of augmented reality based closed loop visual cue training was given to experimental group. Compared to control group, the Walking speed, cadence, stride length of paretic and nonparetic limb, symmetrical walking and SIS-SF score improved 25.1\%, -5.5\%, 29.3\%, 8.3\%, 5.3\% and 29.1\% respectively. The finding of our study supports the beneficial effect of augmented reality based closed loop visual cue training for improving the gait and functional ambulation in stroke patients.

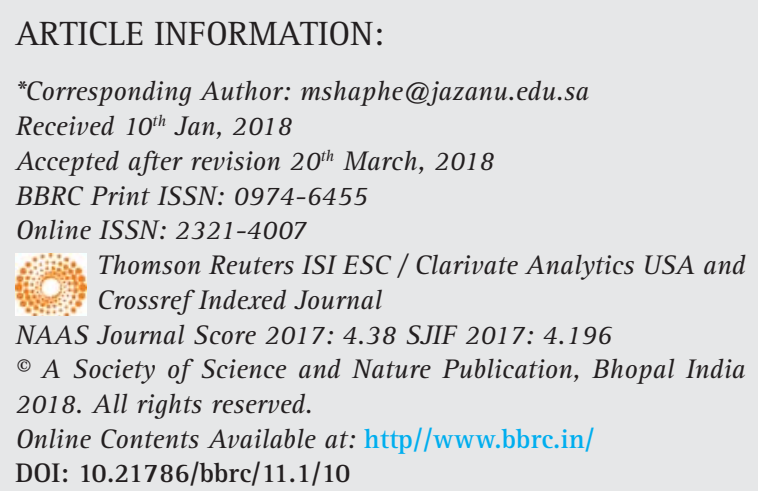




\section{INTRODUCTION}

Stroke is one of the most devastating condition leading to life long morbidly and mortality. The stroke survivors suffer from various sensorimotor impairment of gait and posture leading to moderate to severe disability (Duncan et al 2002). Patients with stroke presents with multiple postural deficits including loss of anticipatory postural reaction, postural sway, uneven weight bearing and inability to maintain upright posture. These deficits increase the risk of falling and ultimately affect the ambulation and activities of daily living (Dickstein et al 2000). Depending upon the severity of stroke most patient recover gait function, though a very small percentage of stroke survivors become community ambulatory (Mackintosh et al 2005). Recent studies proposed that the post stroke impairment of mobility functions, such as, reduced walking speed, asymmetrical weight bearing, unequal step and stride length etc. may be due the inability of the patients to regulate the anticipatory postural reaction (Hill et al 1997). Over and above, the impaired coordination directly affects all the aspects of ambulation including; turning, obstacle avoidance, relative foot placement and velocity regulation, required for independent community ambulation (Roerdink et al 2007). Synthesis of recent literature supports an intensive task specific gait training targeting symmetrical gait pattern may improve ambulatory function (Hollands et al 2012)

The existing evidence suggest that the visual inputs are most import external sensory cues regulating walking and due the impaired sensory inputs the stroke survivors excessively rely on vision to maintain dynamic stability (Kim et al 2012). Gait training incorporating external sensory feedback, which has been found to be very effective in Parkinson's disease (Fuzail et al 2007) have recently begun to be used to investigate functional walking tasks in stroke populations (Bonam et al 2004). In a natural closed loop feedback control system, the physical motion of the body generates the visual cue in response to ambulation and in the absence of movement these cues are not generated (Hollands et al 2010).

The traditional gait training techniques, which are not based on principles of motor control lack requisites in terms of training intensity, duration and enough practice to have any meaningful carryover effect (Rizzo et al 1997). Novel interactive Virtual reality (VR) technologies, creating an immersive environment for stroke patients simulating real-world experience, can help address the limitations posed by traditional approaches (Cikajlo et al 2009). Augmented reality (AR) can improve the implicit knowledge of movement by immersing the desired training regimen in a real-world environment (Azuma et al 2001). A recent systemic review had showed strong scientific evidence supporting the beneficial effects of virtual reality on upper limb motor recovery in stroke patients (Viñas Diz 2016). A recent home based virtual reality training had shown beneficial functional training effects; suggesting that it may be useful as a neurorehabilitation tool (Villiger et al 2017).

To the best of our knowledge, till date, there is no randomized controlled trial demonstrating the efficacy of closed loop visual cues incorporated augmented reality environment on functional gait and community ambulation in stroke patients. Therefore, the aim of this study is to investigate the efficacy augmented reality based closed loop visual cue training on gait and functional ambulation in sub-acute stroke patients.

\section{MATERIAL AND METHODS}

Design: A randomized control trial comparing the effect of proposed intervention with control group was designed. Based on the $80 \%$ power and an alpha value of .05 the sample size calculated to be 14 participants in each group. The subjects were randomly selected based on lottery method and allocated to either closed loop visual cue augmented reality group or to control group receiving traditional gait training. The subjects were selected based on the following inclusion criteria; (a) patients having single episode of stroke (b) onset of stroke must have occurred before months, (c) presence gait deficit due to hemiplegia, (d) patient must be able to walk independently, and (e) must be medically stable without any visual or cognitive defect. Patient having other comorbidity affecting gait function were excluded from the study.

All the subjects recruited in the study underwent a structured one-hour physical therapy session 6 day a week for four consecutive weeks. In addition to this the subjects in the experimental group subjects participated in an additional closed loop visual cues training through a head mounted device (HMD) with partial bodyweight supported treadmill training (Walker et al 2010). The HMD generated visual cues matched to walking symmetry bringing the sensory feedback signals closer to the eyes, making the sensory effect more pronounced, easier to follow and to learn. The closed loop visual cue training was provided every alternate day for 4 weeks.

\section{Outcome measurements}

The following outcome measurements were taken by a therapist at baseline and again after 4 weeks of training. Gait function were measured using GAITRite (GAITRite; CIR system Inc., Havertown, PA, USA). The standard GAITRite walkway contained six sensor pads encapsulated in a rolled-up carpet with an active area of 3.66 


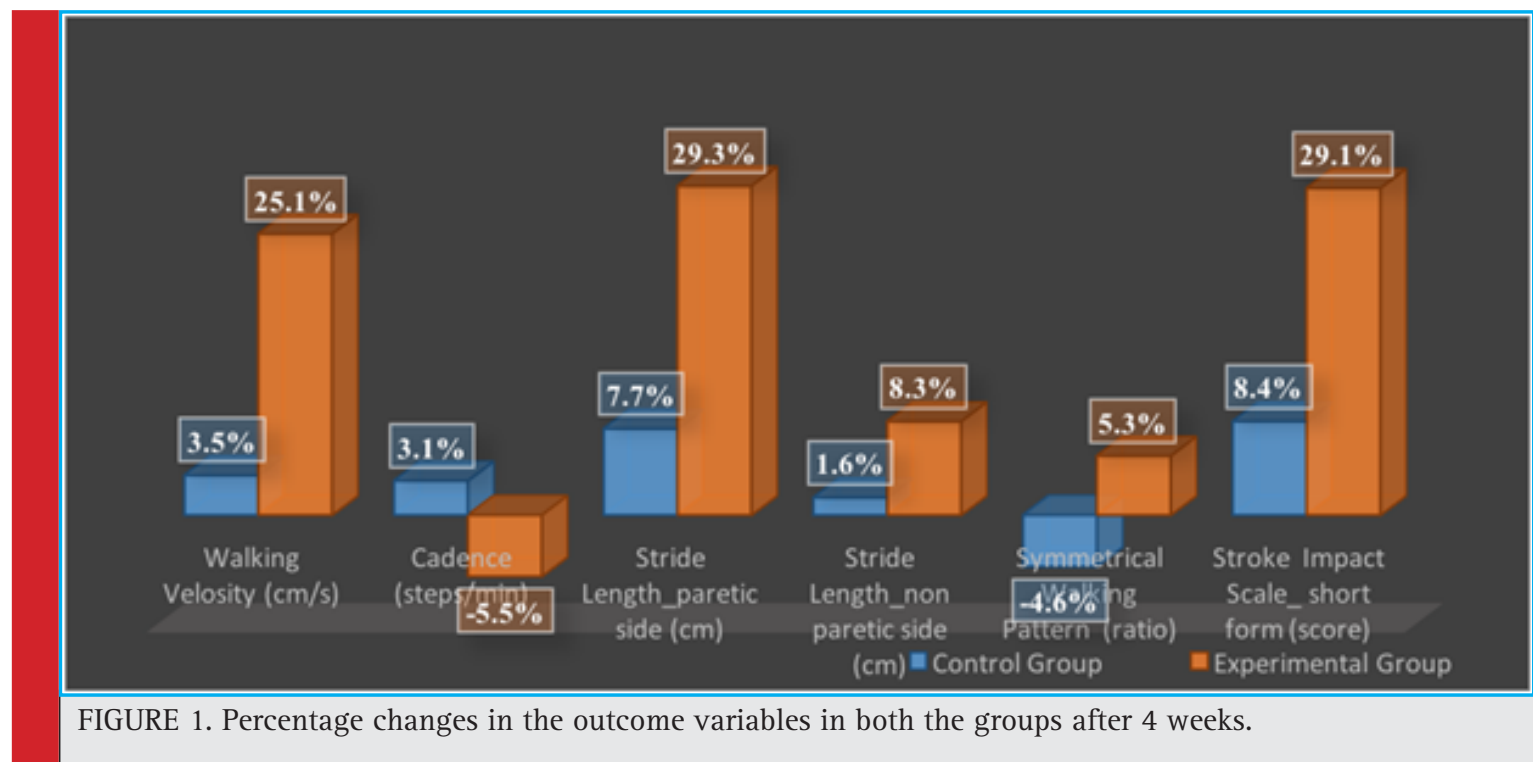

m (length) $0.61 \mathrm{~m}$ (width). GAITRite will be used for measurement of the spatiotemporal parameters, including gait velocity, cadence, step length, and stride length (Mc Donough et al 2001). (Uden et al 2004) investigated the reliability of measurements performed using the GAITRite system as a video-based analysis system and found excellent reliability (Intraclass Correlation Coefficient (ICC) Z0.94).

A short for stroke impact scale (SF-SIS) was used to measure the perceived community participation content, convergent, and discriminant validity (Rachael et al 2016).

\section{RESULTS}

Total 28 patients were enrolled in the study with a mean age of $44.7+12.3$ years and the mean stroke onset was found to be $20.5+9.2$ months. Fourteen subjects were randomly allocated in control and experimental group. Two patients dropped out of the control group and 3 subjects dropped out from the experimental group due to various reason.

An independent t-test was performed to check the uniformity of data which showed that there is no difference in all the selected variable. The results are provided in below mentioned table 1 .

It was found that, in the group getting general physical therapy program, there was an increase of $4 \%$ in walking velocity, 3\% increase in cadence, $8 \%$ improvement in walking symmetry, $2 \%$ increase in stride length of the paretic limb, 5\% decrease in stride length of the non paretic limb and $8 \%$ improvement in the SIS_SF scores. A paired t-test was conducted, with an alpha level of .05 between the baseline and post test scores,

\begin{tabular}{|l|l|l|l|l|l|l|}
\hline \multirow{2}{*}{ Table 1. Baseline Characteristic } & \multicolumn{2}{|c|}{ CG } & \multicolumn{2}{|c|}{ EG } & \multirow{2}{*}{ T } \\
\cline { 2 - 8 } & Mean & SD & Mean & SD & & \\
\hline Age (years) & 46.5 & 13.4 & 42.7 & 11.3 & .727 & 0.475 \\
\hline Stroke Onset (months) & 20.5 & 11.5 & 20.6 & 6.5 & -.012 & 0.991 \\
\hline Walking Velocity (cm/s) & 35.3 & 9.8 & 38.5 & 11.6 & -0.717 & 0.481 \\
\hline Cadence (steps/min) & 71 & 7.0 & 69.8 & 8.8 & 0.357 & 0.725 \\
\hline Stride length-paretic side (cm) & 0.63 & 0.10 & 0.6 & 0.1 & 0.222 & 0.827 \\
\hline Stride length-non paretic side (cm) & 58.5 & 3.2 & 58.8 & 3.0 & -0.247 & 0.808 \\
\hline Symmetrical Walking Pattern (ratio) & 59.8 & 2.4 & 60.5 & 3.2 & -0.529 & 0.603 \\
\hline Stroke Impact Scale_ short form (score) & 20 & 4.3 & 22 & 3.4 & -1.127 & 0.272 \\
\hline CG: Control Group, EG: Experimental Group, SD: Standard deviation & & & & \\
\hline
\end{tabular}




\begin{tabular}{|c|c|c|c|c|}
\hline & $\mathrm{CG}\left(\mathrm{M}_{ \pm} \mathrm{SD}\right)$ & $\mathrm{EG}\left(\mathrm{M}_{ \pm} \mathrm{SD}\right)$ & $\mathrm{T}$ & $\mathrm{p}$ \\
\hline Walking Velocity $(\mathrm{cm} / \mathrm{s})$ & $35.3 \pm 9.8$ & $36.5 \pm 13.5$ & .438 & .670 \\
\hline Cadence (steps/min) & $71 \pm 7$ & $73.2 \pm 8.5$ & 1.116 & .288 \\
\hline Stride length-paretic side $(\mathrm{cm})$ & $58.4 \pm 3.7$ & $59.3 \pm 5.9$ & .557 & .558 \\
\hline Stride length-non paretic side $(\mathrm{cm})$ & $59.8 \pm 2.4$ & $57.1 \pm 5.5$ & 1.817 & .096 \\
\hline Symmetrical Walking Pattern (ratio) & $0.63 \pm 0.1$ & $0.67 \pm 0.13$ & 1.339 & .208 \\
\hline Stroke Impact Scale_ short form (score) & $20 \pm 4.3$ & $22 \pm 5.8$ & 1.301 & .220 \\
\hline
\end{tabular}

Table 3. Result of pairwise comparison of baseline and post test scores of outcome variables in the experimental group.

\begin{tabular}{|c|c|c|c|c|}
\hline & $\mathrm{CG}\left(\mathrm{M}_{ \pm} \mathrm{SD}\right)$ & $\mathrm{EG}\left(\mathrm{M}_{ \pm} \mathrm{SD}\right)$ & $\mathrm{T}$ & $\mathrm{p}$ \\
\hline Walking Velocity $(\mathrm{cm} / \mathrm{s})$ & $38.5 \pm 11.6$ & $48.1 \pm 11.1$ & 4.545 & 0.001 \\
\hline Cadence (steps/min) & $69.8 \pm 8.8$ & $66 \pm 6.9$ & 2.329 & 0.042 \\
\hline Stride length-paretic side $(\mathrm{cm})$ & $58.8 \pm 2.9$ & $63.7 \pm 4.1$ & 3.331 & 0.008 \\
\hline Stride length-non paretic side $(\mathrm{cm})$ & $60.5 \pm 3.2$ & $63.6 \pm 5.5$ & 1.665 & 0.127 \\
\hline Symmetrical Walking Pattern (ratio) & $0.62 \pm 0.14$ & $0.8 \pm 0.07$ & 4.225 & 0.002 \\
\hline Stroke Impact Scale_short form (score) & $22 \pm 3.4$ & $28 \pm 4.6$ & 3.545 & 0.005 \\
\hline
\end{tabular}

which showed that none of the improvement is statistically significant (table 2)

After comparing the baseline and post test scores in the group getting closed loop visual cues training, it was found that there was an increase 25\% in the waking speed, which was found to be statistically significant $(t=4.545, p=.001)$. The cadence showed a decreased of $5 \%$ which was statistically significant $(t=2.329, \mathrm{p}=.042)$. The stride length of the paretic limb increased by $8 \%$ which was found to be statistically significant $(t=3.331$, $\mathrm{p}=.008$ ). Even though, there was an increase of $5 \%$ in stride length of the non paretic limb, it was found to be statistically not significant ( $t=1.665, \mathrm{p}=.127)$.

Compared to control group, the Walking speed, cadence, stride length of paretic and non paretic limb, symmetrical walking and SIS-SF score improved 25.1\%, $-5.5 \%, 29.3 \%, 8.3 \%, 5.3 \%$ and 29.1\% respectively. An independent t-test was utilized to find the effectiveness of both the treatment regimes. The result presented in table 4 suggests that, all the outcome variables showed a statistically significant improvement in the experimental group getting closed loop visual cues.

\begin{tabular}{|c|c|c|c|c|c|c|}
\hline \multirow{2}{*}{ Variables } & \multicolumn{2}{|c|}{ CG } & \multicolumn{2}{|c|}{ EG } & \multirow{2}{*}{$\mathrm{T}$} & \multirow{2}{*}{$\mathrm{p}$} \\
\hline & Mean & SD & Mean & SD & & \\
\hline Walking Velocity $(\mathrm{cm} / \mathrm{s})$ & 36.5 & 13.5 & 48.1 & 11.1 & -2.236 & 0.036 \\
\hline Cadence (steps/min) & 73.2 & 8.4 & 66.0 & 6.9 & 2.213 & 0.038 \\
\hline Stride length-paretic side (cm) & 0.68 & 0.13 & 0.8 & 0.1 & -2.822 & 0.01 \\
\hline Stride length-non paretic side $(\mathrm{cm})$ & 58.3 & 4.6 & 63.7 & 4.1 & -3.012 & 0.007 \\
\hline Symmetrical Walking Pattern (ratio) & 57.1 & 5.5 & 63.6 & 5.5 & -2.858 & 0.009 \\
\hline Stroke Impact Scale_ short form (score) & 22 & 5.8 & 28 & 4.6 & -2.969 & 0.007 \\
\hline
\end{tabular}




\section{DISCUSSION}

The aim of this study was to find the effectiveness a novel method utilizing the augmented reality based closed loop visual cue training to improve the gait function for better community participation in stroke patients. The result of this study has showed an improvement of all gait related variable in the experimental group except stride length of the non paretic limb, which may be due to the relative improvement of the paretic limb's stride length. The result of our study is similar to the study done by (Lee et al 2014). where they have used a virtual reality based postural control for improving the gait function in the stroke patients. Their study showed a significant difference in all gait variables except cadence but in our study we found that the cadence was significantly decreased by $5 \%$. This decrease may be in response to the significant increase in the paretic limb's stride length, which would have caused longer but less steps/minute. We also found an insignificant increase in the non paretic limb's stride length, which is in contrast with the study by (Lee et al 2014). This may be due to the improved motor control of the paretic side, causing leveling of stride length on both the side.

A recent study by XI et al (2017) suggests cortical plasticity by increased activation of cortical regions in stroke survivors as a probable mechanism for better recovery of mobility function associated with virtual reality based training. Another important finding of our study was 29\% significant improvement in symmetrical walking pattern in the experimental group subjects. This finding is similar to the result of the study conducted by (Sami et al 2015) here the symmetrical walking pattern significantly improved due to increased muscle strength and coordination.

As stroke patients are unable to cope with the challenges of varying environmental demands required for community walking due to their impairments. As stroke patients live a relatively sedentary lifestyle any interventions which consistently augments activity level and induces demand on lower limb's motor control may improve the walking pattern (Hendricksan et al 2014). Therefore, the improvement seen in our study may be attributed to the determined efforts of the patients in response to the closed loop visual cues for effectively controlling their lower limbs.

The most noteworthy finding of our study was a substantial improvement in perceived community participation among the subjects closed loop visual cue training group. We saw a $29 \%$ increase in perceived community participation which is in line with the findings of Sami et al 2015. (Warren et al 2016) in their study established a direct relationship between decreased community participation and slower walking speed, which may explain the magnified perception of community participation due to increased walking speed.

\section{CONCLUSION}

The finding of our study supports the beneficial effect of augmented reality based closed loop visual cue training for improving the gait and functional ambulation in stroke patients.

\section{ACKNOWLEDGMENTS}

This research is funded by Sheikh Abdullah Bin Abdul Mohsen Al Tuwaijri Chair for Applied Research in Stroke, Majmaah University, Saudi Arabia. We would like to express our gratitude towards Sheikh Abdullah Al Tuwaijri, and Dr. Khalid Bin Saad Al Muqrin, Rector, Majmaah University, and Deanship of Research, Majmaah University for providing the necessary support and assistance for completing this study.

\section{REFERENCES}

Azuma R, Baillot Y, Behringer R, Feiner S, Julier S, Maclntyre B. (2001): Recent advances in augmented reality. IEEE Comput Graph Appl. 21:34e47.

Bonan IV, Yelnik AP, Colle FM, Michaud C, Normand E, Panigot B, Roth P, Guichard JP, Vicaut E: (2004): Reliance on visual information after stroke. Part II: Effectiveness of a balance rehabilitation program with visual cue deprivation after stroke: a randomized controlled trial. Arch Phys Med Rehabil. 85(2):274-278.

Chi-Ho Lee, Yumi Kim, PT, Byoung-Hee Lee. (2014): Augmented reality-based postural control training improves gait function in patients with stroke: Randomized controlled trial. Hong Kong Physiotherapy Journal:32, 51-57.

Cikajlo I, Matjacic Z. (2009): Advantages of virtual reality technology in rehabilitation of people with neuromuscular disorders. In: Naik Ganesh R, editor. Recent advances in biomedical engineering. Vienna: In-Tech; pp. 301e20.

Dickstein R, Abulaffio N. (2000): Postural sway of the affected and nonaffected pelvis and leg in stance of hemiparetic patients. Arch Phys Med Rehabil; 81:364e7.

Duncan P, Horner R, Reker D, Samsa G, Hoenig H, Hamilton $\mathrm{B}$, et al. (2002): Adherence to post acute rehabilitation guidelines is associated with functional recovery in stroke. Stroke; 33:167e77.

Fuzail Ahmad, Vinay Goel, Leena Dhawan, Mona Maurya. (2007): The Effect of External Sensory Cues on Parkinson's Gait after Deep Brain Stimulation Surgery. IJPOT, Vol.1, No. 1 (2007-01 - 2007-03).

Hendrickson J, K. K. Patterson, E. L. Inness, W. E. Mcllroy, and A. Mansfield, (2014): "Relationship between asymmetry of 
quiet standing balance control and walking post-stroke," Gait and Posture, vol. 39, no. 1, pp. 177-181.

Hill K, Ellis P, Bernhardt J, Maggs P, Hull S: (1997): Balance and mobility outcomes for stroke patients: a comprehensive audit. Aust J Physiotherapy, 43(3):173-180.

Hollands KL, Pelton TA, Tyson SF, Hollands MA, van Vliet PM: (2012): Interventions for coordination of walking following stroke: systematic review. Gait Posture: 35(3):349-359.

Hollands KL, Van Vliet P, Zietz D, Wing A, Wright C, Hollands MA: (2010): Stroke related differences in axial body segment coordination during preplanned and reactive changes in walking direction. Exp Brain Res: 202(3):591-604.

Kim SJ, Krebs HI. (2012): Effects of implicit visual feedback distortion on human gait. Exp Brain Res. 218(3):495-502.

Mackintosh SF, Hill K, Dodd KJ, Goldie P, Culham E. (2005): Falls and injury prevention should be part of every stroke rehabilitation program. Clin Rehabil; 19:441e51.

McDonough AL, Batavia M, Chen FC, Kwon S, Ziai J. (2001): The validity and reliability of the GAITRite system's measurements: a preliminary evaluation. Arch Phys Med Rehabil; 82:419-25.

Michael Villiger, Jasmin Liviero, Lea Awai, Rahel Stoop, Pawel Pyk, Ron Clijsen, Armin Curt, Kynan Eng, and Marc Bolliger (2017) Home-Based Virtual Reality-Augmented Training Improves Lower Limb Muscle Strength, Balance, and Functional Mobility following Chronic Incomplete Spinal Cord Injury. Front Neurol; 8: 635. Published online 2017 Nov 28. doi: 10.3389/fneur.2017.00635

Rachael MacIsaac, Myzoon Ali, Michele Peters, Coralie English, Helen Rodgers, Crispin Jenkinson, et al. (2016): Derivation and Validation of a Modified Short Form of the Stroke Impact Scale. J Am Heart Assoc;5:e03108 doi: 10.1161/ JAHA.115.00310.
Rizzo AA, Buckwalter JG. (1997): Virtual reality and cognitive assessment and rehabilitation: the state of the art. Stud Health Technol Inform.44:123e45.

Roerdink M, Lamoth CJ, Kwakkel G, van Wieringen PC, Beek PJ. (2007): Gait coordination after stroke: benefits of acoustically paced treadmill walking. Phys Ther 87(8):1009-1022.

Sami S. Alabdulwahab, Fuzail Ahmad, and Harpreet Singh. (2015): Effects of Functional Limb Overloading on Symmetrical Weight Bearing, Walking Speed, Perceived Mobility, and Community Participation among Patients with Chronic Stroke. Rehabilitation Research and Practice. Volume (2015), Article ID 241519, 6 pages http://dx.doi.org/10.1155/2015/241519.

Van Uden CJ, Besser MP. (2004): Testeretest reliability of temporal and spatial gait characteristics measured with an instrumented walkway system (GAITRite). BMC Musculoskelet Disorder. 5:13.

Viñas Diz S and M. Sobrido Prieto (2016) Virtual reality for therapeutic purposes in stroke: A systematic review. Neurología; Volume 31, Issue 4, Pages 255-277. https://doi.org/ 10.1016/j.nrleng.2015.06.007

Walker ML, Ringleb SI, Maihafer GC, Walker R, Crouch JR, Van Lunen B, et al. (2010): Virtual reality-enhanced partial body weight supported treadmill training post-stroke: feasibility and effectiveness in 6 subjects. Arch Phys Med Rehabil. 91:115e22.

Warren M1, Ganley KJ2, Pohl PS2. (2016): The association between social participation and lower extremity muscle strength, balance, and gait speed in US adults. Prev Med Rep.7;4:142-7. doi: 10.1016/j.pmedr.2016.06.005.

Xiao X1, Lin Q, Lo WL, Mao YR, Shi XC, Cates RS et al. (2017): Cerebral Reorganization in Sub acute Stroke Survivors after Virtual Reality-Based Training: A Preliminary Study. Behav Neurol.2017:6261479. doi: 10.1155/2017/6261479. Epub 2017 Jun 28. 